\title{
Agôn
}

9| 2021

Rater

\section{Jugements sur les ratages de spectacles et cycle de vie des compagnies}

\section{Daniel Urrutiaguer}

\section{(2) OpenEdition \\ Journals}

Édition électronique

URL : https://journals.openedition.org/agon/8560

DOI : $10.4000 /$ agon. 8560

ISSN : 1961-8581

Éditeur

Association Agôn

Référence électronique

Daniel Urrutiaguer, « Jugements sur les ratages de spectacles et cycle de vie des compagnies », Agôn [En ligne], 9 | 2021, mis en ligne le 16 juillet 2021, consulté le 17 janvier 2022. URL : http:// journals.openedition.org/agon/8560; DOI : https://doi.org/10.4000/agon.8560

Ce document a été généré automatiquement le 17 janvier 2022.

Association Agôn et les auteurs des articles 


\title{
Jugements sur les ratages de spectacles et cycle de vie des compagnies
}

\author{
Daniel Urrutiaguer
}

1 Historiquement, la mission de service public culturel français a été justifiée par l'articulation du soutien étatique à des exigences artistiques de haut niveau, qui ne rencontrent pas spontanément un large public, et à un engagement pour rendre accessibles ces œuvres au plus grand nombre. Même si d'autres doctrines d'action culturelle ont émergé autour des notions de développement culturel territorialisé à partir des années 1970 ou du déplacement plus récent des justifications de l'effort budgétaire vers le respect du multiculturalisme et l'intérêt des retombées économiques des activités artistiques, il s'agit d'un processus d'addition de rhétoriques sans une réelle substitution ${ }^{1}$.

2 La distribution des subventions s'effectue par un processus de délégation des choix à la communauté artistique instituée, proche d'«académies invisibles », en s'appuyant sur les jugements qualitatifs des commissions spécialisées de pairs ainsi que sur le degré de notoriété des directeurs artistiques des compagnies ${ }^{2}$. Les commissions d'experts des Directions régionales des affaires culturelles (Drac) se prononcent d'abord sur une appréciation des mérites artistiques des œuvres, si les spectacles ont été vus par un nombre suffisant de membres afin de permettre une délibération collégiale, tandis que le poids devenu plus important des directions d'établissements culturels dans la composition de ces commissions a induit une prise en considération plus attentive des capacités de diffusion ${ }^{3}$. La mise en concurrence des deux instances de légitimation, la corporation professionnelle et les médias, fait l'objet de tensions entre les échelles d'évaluation de la grandeur dans le monde civique et le monde de l'opinion ${ }^{4}$, dénoncées dès les années 1970 dans le réseau des centres dramatiques nationaux sous la forme d'un « affolement du marché » par le directeur de l'Association technique pour l'action culturelle (ATAC), Louis Cousseau ${ }^{5}$. 
3 Dans ce contexte de circuits de production et de diffusion subventionnés stratifiés selon la cotation corporative et médiatique des équipes artistiques, le cycle de vie des compagnies est en grande partie conditionné par les jugements sur la qualité de leurs créations. Si le travail de la compagnie ne reste pas ignoré par les collectivités publiques, la reconnaissance des qualités de certains spectacles peut s'accompagner d'opinions négatives pour d'autres propositions perçues comme des ratages. Dans quelle mesure cette dénégation de la valeur esthétique de nouvelles œuvres peut se traduire par une dévalorisation monétaire à la suite d'un déclassement de la compagnie ? A quelles conditions cette dépréciation peut être évitée par l'appréhension des sources d'insatisfaction comme des erreurs dans le cadre d'un apprentissage organisationnel ou d'une expérimentation esthétique et non comme une faute de goût, reflet d'un échec artistique?

4 Trois phases du cycle de vie des compagnies sont particulièrement sensibles: les conditions et les obstacles à l'émergence d'une visibilité professionnelle, la postémergence après la reconnaissance plus largement partagée d'un spectacle précédent, le maintien du conventionnement d'une compagnie.

5 L'article s'appuie sur deux études nationales dont j'ai assuré la responsabilité scientifique, l'une sur les territoires et les ressources des compagnies non musicales en France menée sur la période de 2007 à $2009^{6}$, l'autre sur la diffusion de la danse en France de 2011 à $2017^{7}$. Les extraits d'entretiens sont restitués de façon anonyme en respectant la démarche contractuelle proposée lors des entrevues. Des entretiens que j'ai effectués avec Stanislas Nordey en 1998 et François Rancillac en 2010 sont également utilisés afin d'illustrer des faits structurels encore d'actualité.

\section{Comment émerger dans la filière des arts de la scène?}

\section{Les obstacles majeurs à l'émergence}

6 Les œuvres de l'esprit sont caractérisées dans la jurisprudence française par leur originalité, en étant ainsi marquées par l'empreinte de la personnalité de leurs auteurs, et un acte de mise en forme physique. Alors que tout spectacle résulte d'un travail d'équipe qui croise des compétences artistiques, techniques et administratives, les retombées symboliques d'un succès ont tendance à se concentrer sur la personne des créateurs. Il en va de même pour un jugement corporatif d'échec qui peut provoquer une déqualification prématurée et durable de ces derniers auprès de programmateurs ou d'experts des Drac sur-sollicités.

7 La visibilité professionnelle des compagnies théâtrales et chorégraphiques est conditionnée à la création de nouveaux spectacles. Attirer l'attention suppose une capacité à compléter les spectacles du répertoire en tournée par des projets de création sur lesquels échanger avec les programmateurs. Il en résulte une pression à l'accroissement des créations de spectacles et de compagnies, ce qui accentue les difficultés des jeunes équipes artistiques à se distinguer dans la prospection d'opportunités de financement pour le montage de créations et de diffusion pour leur exploitation. Bien que l'enquête sur la diffusion de la danse n'ait pas montré de tendance inflationniste dans les créations de spectacles chorégraphiques déclarés à la Société des auteurs et compositeurs dramatiques (SACD), le maintien d'un haut niveau annuel de nouveaux spectacles (autour de 700 depuis 10 ans) peut être interprété en 
termes de saturation des capacités de circulation dans les réseaux de diffusion, au moins dans ceux des établissements culturels labellisés par l'État et/ou la Région du siège social. Cela conduit les équipes artistiques à tenter de diversifier leurs circuits de diffusion vers d'autres réseaux, notamment les lieux non dédiés au spectacle vivant comme les établissements scolaires ou des maisons des jeunes et de la culture (MJC), des maisons de quartier, des bibliothèques, des musées ${ }^{8}$.

Les obstacles à la reconnaissance artistique durable des compagnies se traduisent par l'intensité et la stabilité des inégalités de diffusion. Ainsi sur l'ensemble de la période 2011-2015, 62\% des spectacles chorégraphiques déclarés à la SACD n'ont été diffusés au plus que cinq fois tandis que $10 \%$ d'entre eux l'ont été plus de 20 fois (avec un maximum de 282 représentations pour Globulus, un spectacle pour l'enfance et la jeunesse chorégraphié par Laurence Salvadori sans subvention) ${ }^{9}$. Ces fortes inégalités relèvent en partie d'une logique d'" appariements sélectifs", analysée par PierreMichel Menger, selon laquelle les équipes se structurent par cooptation entre professionnels de qualité ou de réputation équivalente ${ }^{10}$. Il en résulte une tendance à une segmentation des circuits de production et de diffusion des spectacles selon le niveau de réputation des metteurs en scène ou chorégraphes et des programmateurs. Elle se manifeste notamment par une corrélation positive entre le rayonnement territorial de la diffusion des spectacles au-delà de la région du siège social des compagnies, la probabilité de leur conventionnement étatique et leur degré d'accès au réseau des établissements culturels labellisés ${ }^{11}$.

9 Face à cette segmentation relative des réseaux, la nécessité de montrer des créations pour espérer l'amorçage d'une reconnaissance institutionnelle peut conduire des jeunes équipes à s'engager dans des locations de salles, notamment dans le cadre du festival off d'Avignon, ou des contrats de coréalisation avec un minimum garanti en faveur du propriétaire de petites scènes parisiennes afin de solliciter la venue de programmateurs ou de journalistes culturels. L'échec de fréquentation, à défaut d'une capacité à attirer l'attention des professionnels ciblés, attentifs aux rumeurs corporatives sur les spectacles à voir, peut entraver durablement les perspectives de développement de la compagnie.

\section{Les conditions favorables à une reconnaissance émergente}

10 Une condition favorable à la reconnaissance artistique de jeunes équipes réside dans la nécessité pour les programmateurs de montrer leur capacité à discerner des talents potentiels. La réputation des directions de salles de spectacles repose donc aussi sur leur accompagnement de compagnies encore peu ou non connues. Certains programmateurs s'illustrent par un suivi attentif des spectacles des jeunes créateurs en jouant un rôle pionnier dans leur promotion tandis que les conseillers sectoriels des Drac établissent des calendriers de déplacement des experts des commissions afin d'élargir leur horizon de connaissance des jeunes équipes artistiques.

11 Cette question est devenue plus récemment une préoccupation explicite des collectivités publiques avec l'organisation des Assises de la jeune création (AJC) au cours du premier semestre 2015. Le principe directeur a été d'engager une réflexion corporative décloisonnée autour de six thèmes pour améliorer les conditions d'intégration professionnelle des jeunes artistes : la diversité à favoriser dans l'accès aux formations supérieures, aux moyens de production, à la direction des 
établissements culturels; l'adaptation des formations aux besoins futurs; le repérage des jeunes talents; les mobilités internationales entrante et sortante; l'insertion professionnelle ; les solidarités et transversalités pour un renouvellement durable de la création $^{12}$. Parallèlement à la volonté de mettre en place un "réseau social collaboratif de la jeune création $»^{13}$, un résultat tangible de la consultation a porté sur la reconnaissance d'une nouvelle forme de résidence d'artistes dans la circulaire ministérielle du 8 juin 2016, la "résidence tremplin ». Elle concerne les artistes qui n'ont jamais présenté leur travail dans une structure labellisée et ne sont pas en contrat avec une autre entreprise de production et de diffusion. Tout comme pour les résidences de création, de recherche ou d'expérimentation, une aide monétaire est accordée en complément de la mise à disposition gracieuse de locaux, de la prise en charge de l'hébergement tandis que des résultats du travail entrepris peuvent être montrés à un public.

Dans le cadre de résidences d'artistes, si l'équilibre entre les temps de création, de diffusion et de rapports aux publics est respecté, les tâtonnements ne sont pas considérés comme un ratage, indice d'un manque de maturité, mais comme une dimension de toute expérimentation dans un processus de recherche. Contrairement à une opinion professionnelle répandue selon laquelle les résidences d'artistes seraient la solution optimale pour la démocratisation culturelle, grâce à l'attractivité de la permanence artistique sur un territoire, l'effet le plus probant ne porte pas sur l'élargissement de la base sociodémographique du public local, dont le consentement à se déplacer est de fait influencé par la perception de la réputation des compagnies associées, mais sur un accompagnement dans la maturation artistique et administrative des équipes invitées ${ }^{14}$.

Les résidences d'artistes, le compagnonnage avec des compagnies conventionnées, des dispositifs de soutien spécifique qui sont notamment renforcés en cas de distinction dans des festivals dédiés à la jeune création concrétisent ainsi cet effort public en faveur de la reconnaissance des équipes artistiques émergentes. Cela ne suffit pas en soi, néanmoins, à effacer le cloisonnement des circuits de production et de diffusion. Les appariements entre ces jeunes artistes et des professionnels confirmés reposent, en effet, sur la cooptation. Les écoles supérieures artistiques jouent un rôle de filtration de compétences qui est pris en considération dans la sélection des équipes en résidence par les directions des établissements culturels. Les avantages compétitifs de leurs anciens élèves reposent aussi sur la constitution de réseaux professionnels élargis grâce aux rencontres marquantes avec des enseignants qui peuvent les coopter et leur ouvrir leur carnet d'adresses, comme dans l'exemple significatif de Stanislas Nordey avec Jean-Pierre Vincent quand il avait suivi le cursus de mise en scène au CNSAD au milieu des années $1980^{15}$, ou des professionnels invités sur de courtes durées.

Malgré l'appel des AJC pour favoriser la visibilité d'une plus grande diversité sur les plateaux, les discriminations de genre ou de couleur de peau continuent à entraver les trajectoires professionnelles des femmes et des descendants de l'immigration pour s'engager dans des mises en scène ou des chorégraphies. Les stéréotypes sexués ${ }^{16}$ et chromatiniens ${ }^{17}$ sur les capacités individuelles de direction artistique augmentent la probabilité de rejet durable à la suite des réserves de programmateurs sur la perception de ratages de maquettes ou de spectacles. Certaines directions d'établissements culturels se sont néanmoins engagées dans la déconstruction active de ces préjugés et 
essaient de fluidifier la circulation de ces jeunes compagnies en les accompagnant dans leur processus de recherche.

\section{Quelles trajectoires professionnelles après l'émergence?}

\section{Les obstacles majeurs lors de la post-émergence}

Dans un article polémique publié par Libération au moment du festival d'Avignon en 2001, Jean-Pierre Thibaudat a opposé la figure pionnière et héroïque du directeur de théâtre, tête chercheuse de la décentralisation, à la figure comptable et ternie du programmateur, assimilé à un gérant de la chaîne Sodexo de livraison de plats formatés. Cette logique d'action industrielle s'accompagne d'une logique plus marchande de recherche de " coups ", à la base d'un «théâtre jetable comme un rasoir Bic » :

Le vrai théâtre est fait d'aventures lentes et tâtonnantes. Aujourd'hui, comme les fruits de nos marchés, il est cueilli trop tôt, risque de pourrir prématurément. Un système piégeant pour l'artiste qui signe un premier spectacle, le critique qui le remarque ${ }^{18}$.

En recourant à un vocabulaire économique plus mesuré, on peut mettre en avant la formation de séquences de décisions en termes de "cascades informationnelles" conduisant à des choix conformistes ${ }^{19}$, un phénomène qui est amplifié en situation d'incertitude sur la qualité des œuvres produites, notamment par les jeunes créateurs. Le suivisme des leaders d'opinion s'explique par la croyance partagée qu'ils détiennent un meilleur niveau d'informations par rapport aux connaissances personnelles des protagonistes. Il en résulte des vagues de spéculation sur de jeunes créateurs dont la réputation ne se nourrit pas seulement d'un talent estimé supérieur mais aussi de la cascade des choix d'adoption des directions d'établissements culturels, tenues de réserver une fenêtre de programmation à la découverte des talents. Les metteurs en scène et chorégraphes devenus à la "mode du moment " témoignent volontiers des différences d'accueil lors de leurs tournées soudainement élargies entre les structures qui connaissent le spectacle et s'intéressent à la démarche du créateur, et celles qui remplissent une ligne dans leur cahier des charges ${ }^{20}$.

Ce phénomène de spéculation est certes atténué par les échanges d'informations instaurés par des réseaux de programmateurs nationaux comme les Rencontres interrégionales et interartistiques de diffusion artistique (RIDA), de l'office national de la diffusion artistique (Onda), les Petites Scènes Ouvertes, ou régionaux, comme les Groupes des 20 en Île-de-France ou en Auvergne-Rhône-Alpes par exemple. Cela conduit néanmoins à une forte pression au renouvellement esthétique lors des créations suivantes. La post-émergence devient ainsi une période de transition difficile où les programmateurs à l'affût de nouveautés esthétiques peuvent se détourner des spectacles suivants, jugés ratés par rapport aux promesses de renouvellement des jeunes talents.

18 Cela a été notamment le cas d'une chorégraphe, interrogée dans l'enquête sur les territoires et les ressources des compagnies (cas 25), qui avait étonné le milieu professionnel par sa proposition de mise en mouvement du corps en relation avec des structures sculptées. Elle a obtenu un accueil plus réservé à partir de sa troisième 
création, fondée sur le même principe esthétique. Un autre chorégraphe interrogé dans l'étude sur la diffusion de la danse a formalisé ce problème vécu lors de cette transition très risquée dans la trajectoire de carrière de la façon suivante :

Et d'un autre côté, les gens qui commencent à prendre de l'ampleur on va les jeter parce que finalement ce n'est plus nouveau. Mais qui s'engage pour continuer à développer et à accompagner? Ce que disait X, il m'a dit, un jour, tu es dans la merde parce que pour toi commence la traversée du désert; tu n'es plus un jeune chorégraphe et en plus tu n'es pas encore assez connu, bon courage (Chorégraphe, cas 4).

\section{Quelles voies de durabilité ?}

19 Face à ce risque d'éviction des circuits de production et de diffusion à la suite de réserves sur les ratages de la trajectoire créative par rapport aux attentes de renouvellement esthétique, la stabilisation du positionnement organisationnel et stratégique des jeunes créateurs déjà découverts dépend de leur capacité à fidéliser et élargir un réseau d'établissements culturels partenaires.

Il s'agit d'élargir les soutiens de structures pour contribuer à la coproduction des projets et à l'exploitation des spectacles dans un contexte où l'allongement des séries de représentations, nécessaire au gain en maturation artistique par la mise en relation avec des publics différenciés, se heurte aux capacités de diffusion limitées, marquées par d'importantes inégalités dans le nombre de lieux de programmation par spectacle ${ }^{21}$. L'appréciation de l'articulation esthétique entre la forme et le contenu de l'œuvre compte mais aussi le discours explicitant les intentions de la création, ce qui peut déstabiliser des démarches pragmatiques.

Donc on part juste de ça, et première question : qu'est-ce que tu veux dire vraiment là-dedans ? Qu'est-ce que tu veux chercher comme sens, le propos ? Mais ce que je viens de dire ce n'est pas suffisant ? C'est toujours comme s'il faut pour être reconnu avoir un espèce d'intellect pas possible autour des envies (chorégraphe, cas 1$)$.

L'obtention d'espaces de résidence d'artiste est importante pour permettre de dégager des temps de création ou de recherche avec des conditions matérielles adéquates. Les lieux intermédiaires et indépendants ${ }^{22}$ jouent ici un rôle positif en proposant des formes de coopération à des équipes artistiques, invitées à répéter dans des locaux mis à disposition sans chercher la finalisation d'un spectacle et à présenter à un public local des étapes du processus de recherche si cela peut contribuer à bonifier la démarche artistique. Le cadre de l'économie sociale et solidaire auquel se réfèrent ces lieux offre un terrain propice à des regards bienveillants sur les expérimentations menées sans porter de jugement dépréciatif sur les inaboutissements.

Un tremplin difficilement contournable pour l'élargissement des tournées passe toutefois par la programmation dans des festivals réputés et fréquentés par les programmateurs, qui peuvent bénéficier ainsi de coûts de transaction plus réduits dans un espace-temps circonscrit, ou dans des scènes parisiennes cotées si les dates de représentation ne se situent pas à la fin du premier semestre ${ }^{23}$.

Alors ça reste très jacobin comme vision, mais si tu n'es pas diffusé sur Paris... les programmateurs passent très facilement sur Paris, on l'a bien vu avec les dates au X, j'ai rempli mon carnet pour la saison 18-19 principalement au X (chorégraphe, cas 5) 
prise de risque des metteurs en scène ou chorégraphes lors de spectacles peut se heurter à des frictions concernant les anticipations de succès des programmateurs, dans un contexte de dégradation des conditions de production, marqué par des apports financiers plus limités et volatiles accompagnés de préachats de représentations au coût du plateau. La projection partagée d'un ratage du futur spectacle pour attirer un nombre significatif de spectateurs constitue une source de blocage dans la phase de production et contraint à revoir à la baisse le budget de montage. Cette anticipation de l'environnement professionnel peut être contrecarrée par la réception effective des représentations par la suite, comme l'atteste le succès de la mise en scène de Le pays lointain de Jean-Luc Lagarce par François Rancillac en 2001 par exemple :

Ce spectacle monté "à l'arrache" (car hormis le soutien indéfectible de deux « lagarciens » de la première heure, aucun directeur n'avait voulu coproduire cette pièce labyrinthique, avec onze acteurs, et difficile à lire pour eux), a connu un incroyable succès lors de sa création au Théâtre de la Tempête, à la Cartoucherie, autant du côté du public que de la critique laquelle, du Figaro au Monde et j'en passe, nous a arrosé d'articles dithyrambiques ${ }^{24}$.

Des décalages entre les échelles de jugement des experts des Drac et le positionnement artistique de certaines compagnies peuvent également retarder le conventionnement étatique malgré le succès auprès du public et de certains critiques dramatiques, comme cela a été le cas pour Les Chiens de Navarre jusqu'à l'obtention du statut de compagnie et ensemble au rayonnement national et international (CERNI) en 2017. Leur mise en dérision des codes de la théâtralité était jugée manquer de pertinence artistique par la commission francilienne alors que les pièces de ce collectif étaient programmées dans certaines structures labellisées comme le T2G, Théâtre de Gennevilliers - Centre Dramatique National, sous la direction de Pascal Rambert.

Les frictions peuvent également provenir de l'existence de financements privés même si la démarche de création s'inscrit dans la logique de mission de service public :

- Et donc pour la Drac, il y avait des oppositions d'experts?

- Oui à cause du statut particulier de la compagnie, qui a toujours quasiment été une entreprise privée-publique, puisque moi la compagnie, dès que le spectacle était fabriqué, il était mis en diffusion par X [tourneur privé], ce qui maintenant est en train de devenir une norme (directeur artistique, cas 19).

Enfin, dans une économie tournée de façon systémique vers le soutien à de nouvelles créations par les mécanismes de distribution des subventions publiques et les attentes des programmateurs, certaines reprises de spectacles peuvent faire l'objet de ratage pour l'organisation de tournées.

On l'a jouée six fois [à Avignon], une cinquantaine de programmateurs et aucune date derrière. Voilà c'est une pièce qui a 20 ans. Honnêtement, il y a plein de gens qui ont dit que c'était une belle pièce, que les interprètes étaient magnifiques mais qu'il fallait présenter autre chose du chorégraphe aujourd'hui (administrateur, cas 33).

Le manque de soutien public ou d'intérêt pour des reprises de spectacles est à la source d'une course à la nouveauté, préjudiciable au temps nécessaire de maturation des œuvres scéniques après la première série de représentations.

Moi je préfère privilégier le répertoire parce que la frénésie de la création est quelque chose qui épuise l'artiste et le marché. Ça nécessite de trouver des coproducteurs, du temps de travail pour créer, pour être disponible, pour être créatif et surtout pour moi une pièce elle se bonifie avec le temps. C'est en les 
diffusant, en les tournant qu'elles se trouvent, elles trouvent leur justesse

(chorégraphe, cas 5). questionner sur les conditions de constitution d'une force mémorielle suffisante des spectacles afin de permettre le succès durable de reprises, comme dans le cas de May $B$ qui a accompagné le parcours chorégraphique de Maguy Marin alors que les premières critiques des journalistes culturels ont été dubitatives.

\section{Conventionnement et risques de déclassement}

\section{Les pressions à la mobilité dans la distribution des subventions}

Quatre objectifs ont été fixés dans le programme 131 «Création » de la loi organique relative aux lois de finances (LOLF) qui structure une des trois missions principales du ministère de la Culture avec la préservation et l'enrichissement des patrimoines ainsi que la transmission des savoirs et la démocratisation culturelle. L'objectif $\mathrm{n}^{\circ} 1$ consiste à inciter à l'innovation et à la diversité de la création, et est mesuré par l'indicateur du taux de créations par rapport à la jauge agrégée des lieux de représentation. L'objectif $\mathrm{n}^{\circ} 2$ consiste à donner des bases économiques et professionnelles solides à la création en s'appuyant sur deux catégories d'indicateurs. Le premier type de rapports est décomposé en deux données : la part des fonctions support dans le budget, en visant à limiter la progression des frais fixes afin d'optimiser la part disponible pour les dépenses artistiques; le taux des ressources propres, en encourageant leur hausse. Le deuxième type de ratio concerne la part de la masse salariale artistique dans la masse salariale. L'objectif $n^{\circ} 3$ vise à augmenter la fréquentation des lieux en distinguant la fréquentation totale, celle du public scolaire et celle des jeunes hors parcours scolaire. L'objectif $n^{\circ} 4$ cherche à accroître le rayonnement de la diffusion des œuvres au-delà de la région du siège social des équipes artistiques. Même si ce programme de la LOLF ne précise pas une obligation minimale dans la rotation des compagnies aidées par les collectivités publiques, un objectif pris en considération par les commissions d'experts vise à assurer un degré de renouvellement dans l'attribution des aides.

Si cette rotation ne pose pas de problème majeur pour la distribution des aides à la création, les enjeux sont plus importants pour les décisions de dé-conventionnement. En effet, la suppression d'une subvention pluriannuelle étatique, même si elle est effectuée par paliers, déstabilise à court terme le modèle économique de la compagnie en la rendant plus dépendante des recettes d'activité pour le financement de son budget de fonctionnement. La décision de déclassement peut susciter un effet de levier négatif auprès d'établissement culturels partenaires si leurs directions interprètent ce retrait comme un signal de qualité défaillante. L'articulation entre les créations de spectacles et l'implication dans des dispositifs d'action culturelle pour consolider l'ancrage territorial de la compagnie est rendue ainsi plus compliquée. Les possibilités de mobilité dans les circuits de production et de diffusion stratifiés selon la réputation corporative et médiatique tendent alors à se restreindre pour la compagnie déconventionnée.

De plus, comme le budget du programme «Création» en euros constants est relativement stable, les enveloppes distribuées aux Drac le sont aussi. La décision de création du nouveau label des CERNI en 2016, afin d'offrir une alternative 
d'accompagnement renforcé aux directeurs artistiques qui ne souhaitent pas prendre la tête d'un établissement, n'a pas été accompagnée de moyens supplémentaires significatifs alors que le seuil annuel de subventionnement a été porté de $80000 €$ à 150 000€. Dans ces conditions, la création de flux d'entrée dans le dispositif du conventionnement doit être complétée par des flux de sortie.

\section{La portée des jugements dépréciatifs} d'un manque de renouvellement esthétique à l'issue du parcours artistique qui a été soutenu par les collectivités publiques. Plusieurs compagnies théâtrales ou chorégraphiques ont ainsi fait l'objet d'un dé-conventionnement à la suite de la perception d'un vieillissement de la capacité créative par les commissions d'experts des Drac. Le basculement se produit notamment à l'occasion d'un spectacle jugé raté, en ne répondant pas aux exigences artistiques attendues par la commission, alors qu'il peut continuer à tourner de façon significative dans des établissements culturels.

De même, l'intérêt prêté au soutien de l'émergence peut compliquer la viabilité économique de compagnies qui ont une certaine ancienneté et se sentent concurrencées par les jeunes équipes nouvellement reconnues.

Des compagnies ont du mal à boucler leurs productions alors qu'elles sont reconnues car les programmateurs sont frileux et ont envie de programmer les émergents-tendance (chorégraphe, cas 29).

Ce que je veux dire c'est qu'on est passé de jeune compagnie, que les gens avaient envie d'aider, soutenir, à dinosaure, où le truc est en train de se fossiliser. Et qu'on a été aussi poussé par des nouvelles compagnies qui émergeaient, et que, voilà, on a aussi subi un peu l'usure de l'inscription dans le temps (metteur en scène, cas 17).

Les jugements sur les ratages esthétiques à la source d'une décision de déconventionnement ou de suppression de subvention peuvent porter aussi sur l'illégitimité prêtée à certains objets, qui n'entrent pas dans le champ de l'horizon d'attente institutionnel.

Après j'avais fait les $\mathrm{X}$. C'est une pièce qu'on a beaucoup tournée et qui tourne encore. C'est des soirées d'improvisation finalement, qui ont été très critiquées par la profession. J'ai eu une coupe drastique de subvention en Région à cause de cette pièce parce que ce n'était pas de l'art. C'était en Y qu'on m'a coupé les subventions, alors qu'on avait beaucoup de diffusion. Et après j'ai créé une pièce plus écrite, avec une belle écriture, donc j'ai été ré-accepté par les institutions (chorégraphe, cas 4).

Une évolution du positionnement artistique vers une attitude de "franc-tireur $»^{25}$ conduit à poursuivre un travail sans le soutien des professionnels intégrés dont la légitimité peut être contestée avec une virulence aigrie comme dans cet exemple :

Je ne corresponds pas, ni à la gaytitude de 30 ans portant des strings et ventres plats, ni à une idée plus institutionnelle, parce que j'ai choisi de travailler sur des objets plastiques, parce que j'ai choisi de les emmerder (chorégraphe, cas 3).

La légitimité de son déclassement institutionnel est ici dénoncée par ce chorégraphe en s'appuyant sur la projection d'une image fantasmée de la mode chorégraphique contemporaine, qui serait dominée par les canons esthétiques d'une jeune communauté professionnelle gay.

37 L'expertise artistique de la commission d'une Drac qui se prononce en faveur d'une décision de dé-conventionnement à la suite d'un jugement plus ou moins consensuel 
sur le ratage esthétique du ou des derniers spectacles peut entrer en conflit avec la vision politique d'élus locaux, plus attentifs au travail d'ancrage territorial de la compagnie. L'implication de l'équipe artistique esthétiquement désavouée dans les dispositifs d'action culturelle, notamment en faisant participer une partie de la population, répond aux attentes politiques locales de consolidation de la cohésion sociale. S'ils ont une influence nationale, les élus concernés peuvent alerter le cabinet du ministère de la culture sur le caractère contre-productif de la mesure en obtenant une écoute attentive. Une pression ministérielle auprès de la direction de la Drac conduit dans certains cas à mettre en échec, au moins à court terme, la proposition de déclassement de la commission. Les jugements sur les ratages esthétiques peuvent ainsi faire aussi l'objet de ratage politique...

\section{Pour conclure}

38 La pluralité des logiques d'action et d'évaluation dans le monde des arts de la scène subventionnés conduit à mettre en perspective les jugements sur les ratages de spectacles. Il peut y avoir des convergences mais aussi des écarts entre les appréciations des commissions d'experts des Drac sur les mérites artistiques, les attentes en termes de politique culturelle des collectivités territoriales, la réception critique des journalistes culturels, ainsi que des spectateurs, qui sont par ailleurs animés par des horizons d'attente diversifiés.

Le cycle de vie des compagnies est d'abord dépendant des jugements sur la qualité des spectacles nouvellement créés par l'expertise professionnelle des pairs auxquels l'État a délégué une autonomie de choix, ainsi que des regards portés par les médias influents à une échelle nationale. Dans un contexte systémique bien plus favorable aux créations de spectacles qu'à leur reprise, les circuits de production et de diffusion sont structurés par une stratification, qui s'appuie sur une logique concurrentielle d'appariements sélectifs en fonction de degrés de réputation similaires. La mobilité entrante et sortante dans ces circuits est ainsi sensible aux jugements sur les réussites et les échecs de spectacles. La logique de coopération à laquelle se réfèrent davantage les jeunes générations d'artistes induit néanmoins un regard plus compréhensif sur les ratages, appréhendés comme une dimension inhérente aux processus d'apprentissage et de recherche-expérimentation. Les résidences de création, de recherche et d'expérimentation offrent un cadre potentiellement intéressant pour œuvrer dans ce sens si les cooptations s'élargissent au-delà du cercle des anciens élèves des écoles artistiques supérieures et si les temps de recherche sont suffisants.

\section{BIBLIOGRAPHIE}

BECKER, Howard, Les mondes de l'art, Paris, Flammarion, 1988. 
BICKHCHANDANI, Sushil, HIRSHLEIFER, David et WELCH, Ivo, “A Theory of Fads, Fashion, Custom and Cultural Change as Informational Cascades", Journal of Political Economy; vol. 100, n5, 1992, p. 992-1006.

BOLTANSKI, Luc, et THEVENOT, Laurent, De la Justification : les économies de la grandeur, Paris, Gallimard, 1991.

CHALAYE, Sylvie, Race et théâtre. Un impensé politique, Arles, Actes Sud-Papiers, 2020.

COUSSEAU, Louis, « Etude du marché théâtral dans les établissements membres de l'ATAC », ATAC Infos, n63, décembre, 1974, p. 24-25.

MENGER, Pierre-Michel, « Les politiques culturelles. Modèles et évolutions », in Philippe Poirrier [dir.], Pour une histoire des politiques culturelles dans le monde : 1945-2011, Paris, La Documentation Française, 2011, p. 465-477.

MENGER, Pierre-Michel, Portrait de l'artiste en travailleur. Métamorphoses du capitalisme, Paris, Éditions du Seuil, 2002.

MINISTERE DE LA CULTURE ET DE LA COMMUNICATION, Mesures en faveur de la formation, de l'insertion, des conditions de vie et de la diversité des jeunes créateurs, 30/06/2015 [en ligne] URL : https://www.culture.gouv.fr/Presse/Archives-Presse/Archives-Dossiers-depresse-2011-2018/Annee-2015/Mesures-en-faveur-de-la-formation-de-l-insertion-desconditions-de-vie-et-de-la-diversite-des-jeunes-createurs [site consulté le 27/07/2020].

MINISTERE DE LA CULTURE ET DE LA COMMUNICATION, Assises de la Jeune Création, Conférence de lancement 17 avril 2015 [en ligne], URL : https://www.culture.gouv.fr/Presse/Archives-

Presse/Archives-Dossiers-de-presse-2011-2018/Annee-2015/Dossier-de-presse-des-Assises-de-laJeune-Creation [Site consulté le 27/07/2020].

PRAT, Reine, Arts du spectacle. Pour l'égal accès des femmes et des hommes aux postes de responsabilité, aux lieux de décision, aux moyens de production, aux réseaux de diffusion, à la visibilité médiatique, Paris, Ministère de la Culture, 2009.

THIBAUDAT, Jean-Pierre, « Le temps des programmateurs », Libération, 9/07/2001.

URFALINO, Philippe, « Les politiques culturelles : mécénat caché et académies invisibles », L'année sociologique, 1989, n³9, p. 81-109.

URRUTIAGUER, Daniel, La diffusion de la danse en France. 2011-2017, Paris, Onda, 2019, in Onda [en ligne], URL : http://www.onda.fr/wp-content/uploads/2017/03/ Onda_etudedanse_complet.pdf [site consulté le 27/07/2020].

URRUTIAGUER, Daniel, « A avaliação das companhias de teatro pelo Ministério da Cultura em França », Análise Social, 2014, vol. 49, n² 213, p. 906-923.

URRUTIAGUER, Daniel, "Performing Arts Programming Strategies and Demand. The case of the Blanc-Mesnil Forum (France)”, International Journal of Arts Management, 2014, vol. 17, n¹, p. 31-42. URRUTIAGUER, Daniel, HENRY, Philippe, et DUCHENE, Cyril, « Territoires et ressources des compagnies en France », Cultures Etudes 2012-1, 2012.

URRUTIAGUER, Daniel, RANCILLAC, François, « Un binôme pour entrer dans le théâtre (entretien) ", Registres, n¹5, 2011, p. 60-69. 


\section{NOTES}

1. Pierre-Michel Menger, "Les politiques culturelles. Modèles et évolutions ", dans Philippe Poirrier [dir.], Pour une histoire des politiques culturelles dans le monde: 1945-2011, Paris, La Documentation Française, 2011, p. 476.

2. Philippe Urfalino, «Les politiques culturelles : mécénat caché et académies invisibles ", L'Année sociologique, 1989, n 39, p. 81-109.

3. Daniel Urrutiaguer, «A avaliação das companhias de teatro pelo Ministério da Cultura em França " (L'évaluation des compagnies théâtrales par le Ministère de la Culture en France), Análise Social, 2014, vol. 49, n² 213, p. 906-923.

4. Luc Boltanski et Laurent Thévenot, De la Justification: les économies de la grandeur, Paris, Gallimard, 1991.

5. Louis Cousseau, «Etude du marché théâtral dans les établissements membres de l'ATAC ", ATAC Infos, n63, décembre, 1974, p. 24-25.

6. Daniel Urrutiaguer, Philippe Henry, Cyril Duchêne, « Territoires et ressources des compagnies en France ", Cultures Etudes 2012-1, 2012.

7. Daniel Urrutiaguer, La diffusion de la danse en France. 2011-2017, Paris, Onda, 2019, in Onda [en ligne], URL : http://www.onda.fr/wp-content/uploads/2017/03/ Onda_etudedanse_complet.pdf [site consulté le 27/07/2020].

8. Daniel Urrutiaguer et al., "Territoires et ressources des compagnies en France », op.cit., p. 2.

9. Daniel Urrutiaguer, La diffusion de la danse en France. 2011-2017, op.cit., p. 11-12.

10. Pierre-Michel Menger, Portrait de l'artiste en travailleur. Métamorphoses du capitalisme, Paris, Seuil, 2002, p. 44.

11. Daniel Urrutiaguer et al., "Territoires et ressources des compagnies en France », op.cit., p. 5.

12. Ministère de la Culture et de la communication, Assises de la Jeune Création, Conférence de lancement 17 avril 2015 [en ligne], URL : https://www.culture.gouv.fr/Presse/Archives-Presse/ Archives-Dossiers-de-presse-2011-2018/Annee-2015/Dossier-de-presse-des-Assises-de-la-JeuneCreation [Site consulté le 27/07/2020]

13. Ministère de la Culture et de la Communication, Mesures en faveur de la formation, de l'insertion, des conditions de vie et de la diversité des jeunes créateurs, 30/06/2015 [en ligne] URL : $\quad$ https://www.culture.gouv.fr/Presse/Archives-Presse/Archives-Dossiers-depresse-2011-2018/Annee-2015/Mesures-en-faveur-de-la-formation-de-l-insertion-desconditions-de-vie-et-de-la-diversite-des-jeunes-createurs [site consulté le 27/07/2020].

14. Daniel Urrutiaguer, "Performing Arts Programming Strategies and Demand. The case of the Blanc-Mesnil Forum (France)”, International Journal of Arts Management, 2014, vol. 17, n¹, p. 31-42.

15. Entretien avec Stanislas Nordey au TGP de Saint-Denis, le 3/09/1998.

16. Reine Prat, Arts du spectacle. Pour l'égal accès des femmes et des hommes aux postes de responsabilité, aux lieux de décision, aux moyens de production, aux réseaux de diffusion, à la visibilité médiatique, Paris, Ministère de la Culture, 2009.

17. Sylvie Chalaye, Race et théâtre. Un impensé politique, Arles, Actes Sud-Papiers, 2020.

18. Jean-Pierre Thibaudat, «Le temps des programmateurs », Libération, 9/07/2001.

19. Sushil Bickhchandani, David Hirshleifer, et Ivo Welch, "A Theory of Fads, Fashion, Custom and Cultural Change as Informational Cascades", Journal of Political Economy, vol. 100, n5, 1992, p. 992-1006.

20. Par exemple, lors de mon entretien du 3/09/1998, Stanislas Nordey a évoqué cette perception à la suite des premières grandes tournées de La dispute de Marivaux et La légende de Siegfried, un spectacle pour enfants, consécutives à son intégration dans le réseau de diffusion du CDNEJ Heyoka de Sartrouville. « Si certains programmateurs ont une sincère envie de nous recevoir [...], d'autres ont une stratégie de catalogue qui doit inclure le jeune metteur en scène à la mode du moment ». 
21. Daniel Urrutiaguer, La diffusion de la danse, op.cit., p. 11.

22. Voir la cartographie des signataires de la Charte Nationale des Lieux Intermédiaires et Indépendants (CNLII) élaborée en 2014 : https://cnlii.org/qui-sommes-nous/cartographie.

23. Comme les programmations se préparent 12 à 18 mois en avance, les représentations de spectacles à Paris en mai ou juin retiennent beaucoup moins l'attention des directions des établissements culturels.

24. Daniel Urrutiaguer, François Rancillac, « Un binôme pour entrer dans le théâtre (entretien) », Registres, $\mathrm{n}^{\circ} 15,2011$, p. 63.

25. Howard Becker, Les mondes de l'art, Paris, Flammarion, 1988, p. 242-254.

\section{RÉSUMÉS}

Les gains de réputation corporative et médiatique sont impulsés par des jugements positifs des experts professionnels sur les créations dramatiques et scéniques. L'article propose d'analyser les effets des opinions professionnelles sur des ratages de spectacles à différents moments clés du cycle de vie des compagnies, qui peuvent entraîner un déclassement des créateurs, ou être appréhendés, de façon plus compréhensive, comme un apprentissage par essais et erreurs dans le cadre de la recherche-expérimentation. En s'appuyant sur des enquêtes nationales sur les compagnies théâtrales et chorégraphiques coordonnées par l'auteur, l'attention est plus spécifiquement centrée sur l'émergence, marquée par une faible visibilité professionnelle, les risques d'éclatement de bulle spéculative lors de la post-émergence et les propositions de déconventionnement.

\section{INDEX}

Mots-clés : cycle de vie, jugement, réputation, spéculation, circuit, résidence.

\section{AUTEUR}

\section{DANIEL URRUTIAGUER}

Professeur en économie et esthétique du théâtre, Alliance Sorbonne, Université de la Sorbonne Nouvelle, Institut de Recherche en Études Théâtrales 